\title{
BMJ Open Functional MRI-guided microsurgery of intracranial arteriovenous malformations: study protocol for a randomised controlled trial
}

\author{
Bing Zhao, Yong Cao, Yuanli Zhao, Jun Wu, Shuo Wang
}

To cite: Zhao B, Cao Y, Zhao Y, et al. Functional MRIguided microsurgery of intracranial arteriovenous malformations: study protocol for a randomised controlled trial. BMJ Open 2014;4:e006618.

doi:10.1136/bmjopen-2014006618

- Prepublication history for this paper is available online. To view these files please visit the journal online (http://dx.doi.org/10.1136/ bmjopen-2014-006618).

Received 12 September 2014 Accepted 2 October 2014

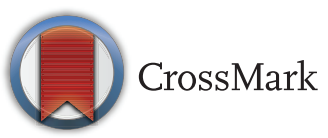

Department of Neurosurgery, Beijing Tiantan Hospital, Capital Medical University; Center of stroke, Beijing institute for brain disorders; Beijing Key Laboratory of Translational Medicine for Cerebraovascular Disease; China National Clinical Research Center for Neurological Diseases, Beijing, China

Correspondence to Dr Shuo Wang; captain9858@vip.sina.com

\section{ABSTRACT}

Introduction: Intracranial arteriovenous malformations (AVMs) are associated with high morbidity and mortality. Modern microsurgery has improved the results of surgical treatment of AVMs; however, the treatment of AVMs, particularly eloquently located AVMs, still carries a high risk. Functional MRI (fMRI) has been reported to be used for the preoperative evaluation of AVMs in small case series. The purpose is to identify the utility and efficacy of fMRI-guided microsurgery of AVMs in a large randomised controlled trial.

Methods and analysis: The study is a prospective, randomised controlled clinical trial. This study will enrol a total of 600 eligible patients. These eligible patients will be randomised to the standard microsurgery group and the fMRI-guided microsurgery group in a 1:1 ratio. Patient baseline characteristics and AVM architecture and characteristics will be described. In the fMRI-guided group, fMRI mapping of an eloquent cortex in all AVMs will be identified. Surgical complications and outcomes at pretreatment, post-treatment, at discharge and at 1 month, 3-month and 6-month follow-up intervals will be analysed using the modified Rankin Scale (mRS). This trial will determine whether fMRI-guided microsurgery could improve outcomes in patients with AVMs and also identify the safety and efficacy of fMRI-guided microsurgery.

Ethics and dissemination: The study protocol and written informed consent were reviewed and approved by the Institutional Review Board of Beijing Tiantan Hospital Affiliated to Capital Medical University (ky2012-016-02). Study findings will be disseminated in the printed media.

Trial registration number: ClinicalTrials.gov NCT01758211.

\section{BACKGROUND}

Intracranial arteriovenous malformations (AVMs) are estimated to have a prevalence of 1.4-4.3\%. ${ }^{1}$ Intracranial haemorrhage and epilepsy are the main clinical presentations in patients with AVMs. The annual rehaemorrhage rate of AVMs varies from $6 \%$ to $15.8 \%,{ }^{2}$ but they are associated with a morbidity rate as high as $35 \%$ and mortality rate of $15 \%$ over long-term follow-up. ${ }^{3-6}$ The treatment aim is solely to prevent haemorrhage and rehaemorrhage from AVMs. Current treatment options include medical management, microsurgical treatment, endovascular embolisation, radiosurgery and a combination of multimodal therapy. ${ }^{7}$ Medical management alone may be superior to medical management with interventional therapy for the prevention of death or stroke in patients with unruptured AVMs in the 'A Randomized trial of Unruptured Brain AVMs' (ARUBA) study; ${ }^{8}$ however, there remains a controversy regarding case-selection bias and short-term follow-up in this study.

In clinical practice, optimal treatment should balance the natural history of AVMs against the risk of treatment. ${ }^{9}$ The natural history of AVMs remains unclear, and microsurgery remains the standard treatment of accessible AVMs. ${ }^{5}$ Microsurgical treatment is immediately warranted for patients with progressive neurological deficits caused by haemorrhage $;^{10}$ however, this treatment to prevent haemorrhage itself carries risks. Although modern microsurgery has been reported to improve the overall outcome in patients with AVMs in our previous studies, ${ }^{1-13}$ the treatment of AVMs in an eloquent cortex is always challenging.

Functional MRI (fMRI) can identify a potential functional imaging of the eloquent cortex. ${ }^{14}$ It can also be utilised accurately to localise lesions in an eloquent cortex and to reduce the morbidity of lesions. ${ }^{15}$ Small case series have reported that fMRI is a valuable tool in the preoperative evaluation with AVMs. ${ }^{14-24}$ A review of the role of fMRI also suggested that fMRI could be useful to aid in the decision-making of treatment of AVMs. ${ }^{25}$ However, fMRI signals in a perilesional eloquent cortex were reduced, whereas these signals were normal or increased in 
homologous regions. ${ }^{21}$ These changes could potentially influence the evaluations of surgery. Moreover, standard tasks and a definitive analysis of fMRI data are required to be achieved before drawing a definitive conclusion. Therefore, this large, randomised clinical trial will identify the safety and efficacy of fMRI-guided microsurgery of AVMs.

\section{STUDY OBJECTIVE}

The key purpose of this study is to determine whether fMRI-guided microsurgery could improve overall outcomes in patients with AVMs, and to identify fMRI mapping of an eloquent cortex in a substantial cohort of patients with AVMs. We will assess the safety and efficacy of fMRI-guided microsurgery.

\section{METHODS AND ANALYSIS}

\section{Study designs}

The study is a prospective, assessor blinded, randomised controlled clinical trial. Eligible patients with AVMs undergoing surgery will be randomised to the standard microsurgery group and the fMRI-guided microsurgery group in a 1:1 ratio. The study protocol was performed at Tiantan hospital (China National Clinical Research Center for Neurological Diseases), which is the largest neurosurgical centre in China. All patients with AVM aneurysms will be treated by the senior author (SW). A diagram of the protocol is presented in figure 1 .

\section{Study participants}

Patient baseline characteristics (age, sex and neurological function), AVM architecture (feeding arteries, nidus, draining veins) and characteristics (size and location) will be provided. Treatment decision is made on the basis of AVM characteristics (the size, location and Spetzler-Martin grade ${ }^{26}$ ). Surgical treatment is preferably considered for patients who have a history of haemorrhage or seizure. Treatment modalities for AVMs and randomised methods will be informed to the patient and family. Eligible patients will be included before the written informed consent is obtained. These patients can withdraw from the study at any time.

\section{Inclusion criteria}

- Patient age ranging from 12 to 60 years.

- Definitive diagnosis of AVMs

- No contraindications to perform fMRI examination

- Informed consent.

\section{Exclusion criteria}

- Patients initially undergoing endovascular and radiosurgery.

- Patients undergoing surgical treatment within 1-month of initial haemorrhage.

- Patients undergoing emergency removal of intracranial haematomas due to AVMs.

- Contraindications for general anaesthesia and surgery.

- Pregnancy and breastfeeding.

\section{Randomisation and blinding}

Eligible patients will be randomised to the standard microsurgery group and the fMRI-guided surgery group in a 1:1 ratio. Stratified blocked randomisation and minimisation methods are employed. Stratification factors include AVM location, where AVMs are located within or in an eloquent cortex, and the Spetzler-Martin grade. It is impossible to blind the participants and surgeons, and only outcome assessors are blinded. These assessors are
Figure 1 Diagram of the study protocol. AVMs, arteriovenous malformations; fMRI, functional MRI; DSA, digital subtraction angiography; mRS, modified Rankin Scale.

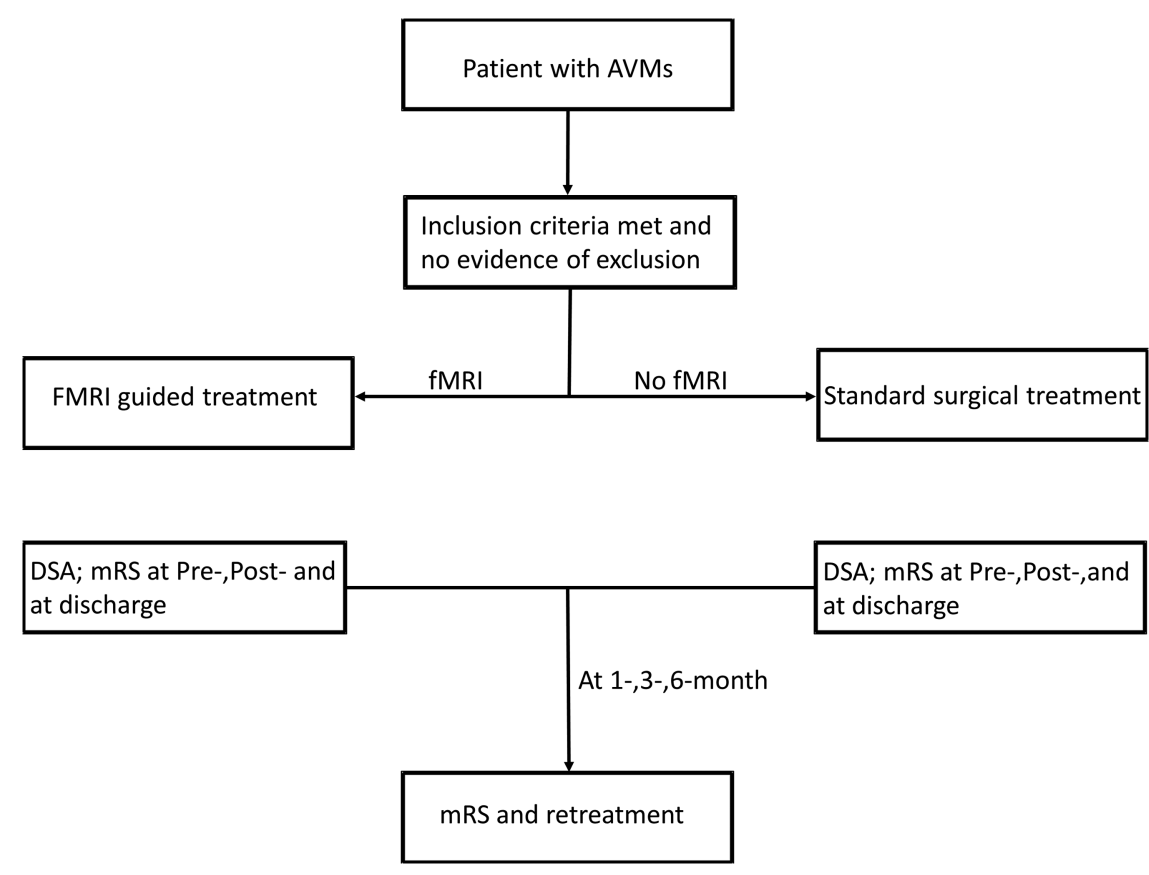


not involved in patient management. The allocation sequence will be generated by an independent third party of the National Center for Cardiovascular Diseases of the People's Republic of China, and patients treated with fMRI-guided microsurgery will be assigned by the third party. In order to prevent bias, the patient randomisation process will be performed by the third party.

\section{Standard microsurgery group}

Patients in the standard group will receive cerebral angiography and MRI before surgical treatment. The craniotomy will be performed within the cerebrovascular team. Standard microsurgical treatment includes temporary clipping of the feeding arteries, careful dissection of the AVM from the adjacent brain, and complete or incomplete resection of the AVM. ${ }^{11}{ }^{12}$ Any techniques for motor evoked potential (MEP) and somatosensory evoked potential (SSEP) can be used for intraoperative monitoring. Intraoperative ultrasound and indocyanine green videoangiography can also be used to guide the treatment of AVMs as needed.

\section{FMRI-guided microsurgery group}

If patients are randomised to the fMRI-guided group, they will perform the fMRI scans within 1 week before surgery. Blood-oxygen-level dependent (BOLD), diffusion tensor imaging (DTI) and time-of-flight MR angiography (TOF-MRA) will be performed. ${ }^{22} 2427$ Different fMRI tasks, such as language, motor and visual tasks, will be respectively employed on the basis of AVM location. These tasks will be performed as follows: (1) language stimulation paradigms acquired by both verb generation and picture naming; (2) motor stimulation paradigms acquired by finger tap movement; (3) visual stimulation acquired by the black-and-white checkerboard. BOLD-fMRI and DTI are automatically fused with threedimensional (3D)-TOF MRA images. The distance between the lesion and the eloquent cortex will be measured. The distance measurement was obtained from axial, sagittal and coronal imaging of MRA. AVM architecture, including the feeding artery, nidus and draining vein, will be acquired by the 3D MRA.

The treatment planning station will be situated based on the fMRI data. Craniotomy will be performed according to the planning. With the brain cortex exposed after craniotomy, the feeding arteries, nidus and draining veins of the AVM will be identified with the navigator's probe. Then a microsurgical resection of AVMs will be performed just like in the standard treatment group.

The fMRI-guided microsurgery will be discontinued if there is a medical necessity or at the request of the participant. This could occur if the patient requires emergency surgery for intracerebral haemorrhage during the study period or if the participant withdraws from the study at any time and for any reason. Investigators will collect data in detail at the time of study randomisation. Adherence to treatment allocation will be monitored in the monthly reports, which originate from the data monitoring centre (National Center for Cardiovascular Diseases of the People's Republic of China). All microsurgical techniques and equipment in both treatment groups will be provided as needed. Any concomitant medication is permitted in the context of the study.

\section{Outcome measures}

The primary outcome is the removal rate of AVMs (complete, near-complete and incomplete) according to the postoperative evaluation of cerebral angiography. The primary outcome is the postoperative surgical complication, neurological function and retreatment. Surgical complications include intracranial infection, epilepsy, rehaemorrhage and cerebral infarction. Patient baseline neurological function and outcomes at pretreatment, post-treatment, at discharge and at 1-month, 3-month and 6-month follow-ups are measured using the modified Ranking Scale (mRS). A schematic diagram is showed in figure 1 . An mRS of $0-2$ is identified as a favourable outcome, and a score of $3-6$ as an unfavourable outcome.

\section{Sample size}

According to the current literature and our previous study, ${ }^{11} \quad 12 \quad 282^{29}$ favourable outcomes (mRS of $0-2$ ) account for approximately $80 \%$ in patients with AVMs after the standard microsurgery. We hypothesise that the overall favourable outcome in the fMRI-guided group could be $90 \%$. A significant difference between the standard microsurgery group and the fMRI-guided group is $10 \%$. A sample size of 300 in the standard group and of 300 in the fMRI group will be included with a two-sided significance level of $5 \%$, a power of $80 \%$, assuming that $10 \%$ of the included patients will be lost to follow-up.

\section{Statistical analysis}

Data verification will be undertaken in $20 \%$ of all cases to assess the accuracy of data recording. Data are given as the mean and SD for continuous variables or frequency for categorical variables. Significances in clinical variables and outcomes between two groups are analysed using the $\mathrm{t}$ test, $\chi^{2}$ test, Fisher's exact test or the Mann-Whitney $\mathrm{U}$ test. Associations of variables will be identified using a univariate and multivariate analysis. Statistical analysis will be performed at the National Center for Cardiovascular Diseases of the People's Republic of China.

\section{Data management}

Data are prospectively collected using an electronic case report form (eCRF) through a study website using a login and password. Neurological conditions and complications related to surgical procedure will be carefully monitored during the whole period of follow-up. These data safety will be monitored at the National Center for Cardiovascular Diseases of the People's Republic of China during the clinical trial. The monthly auditing, data quality and statistical analysis are managed by a 
third party who shall be responsible for notifying the principal investigator and Institutional Review Board of Beijing Tiantan Hospital of any issues that arise. Any serious adverse events will be reported to the Institutional Review Board. Recommendations will be forwarded to the principal investigators for a review of risk and benefit. The Institutional Review Board of the hospital shall have access to these interim results and make the final decision to terminate the trial.

\section{Duration of the study}

This study will enrol eligible 600 patients with AVMs treated with microsurgical treatment in Tiantan hospital (China National Clinical Research Center for Neurological Diseases). Study recruitment commenced in February 2013. The final patient recruitment will end in June 2015.

\section{ETHICS AND DISSEMINATION}

The study was granted approval as a key program by the National Science and Technology Support Program in 2012. The Original Protocol for a prospective multicentre study was registered in December 2012. To reduce surgery bias and fMRI bias in the multicentre, the study protocol was only performed at Tiantan Hospital. Patients allocated to the fMRI-guided treatment group will perform fMRI examinations. Those patients who were implanted with medical devices such as pacemakers will be excluded. Other unexpected risks of fMRI examination will be monitored. A minor amount of brain shift exists in neuronavigation, ${ }^{30}$ but it does not influence the surgical procedure. This study adheres to good clinical practice and ethical principles described in the Declaration of Helsinki and 'Human Biomedical Research Ethical Issues and Policy Guidance'. Written informed consent will be obtained from each participant or their family members. If the patient is less than 18 years old, the informed consent will be obtained from his or her guardian.

This project is supervised by the Ministry of Health of the People's Republic of China. Professor Shuo Wang is the principal investigator. Data interpretation and dissemination of study results will be managed under his direct supervision. The final results will be disseminated in December 2015. The study findings will be disseminated in the printed media.

\section{DISCUSSION}

With advances in neuroimaging, neuromonitoring and microsurgical techniques, modern microsurgery has improved outcomes in patients with AVMs over the past decades. Surgical treatment of AVMs located within or near the eloquent cortex is associated with high morbidity. ${ }^{31}$ FMRI could provide detailed functional imaging data into the navigational guidance system, which can help guide the microsurgery of AVMs. Only a few studies have reported that the integrated preoperative and intraoperative functional navigation was used to resect an AVM located in the primary motor cortex. The first large randomised controlled study mainly focused on outcomes of eloquently located AVMs. FMRI can safely be used for the microsurgery of eloquently located AVMs, and fMRI-guided microsurgery could improve the overall outcomes in patients with AVMs.

Contributors SW obtained research funding and was the principal investigator of the study. YC, YZ and SW developed this study protocol. BZ, YC, YZ, JW and $S W$ all participated in the final design of the study.

Funding This work was funded by the National Science and Technology Support Program of China (grant numbers: 2011BAI08B08, 2012CB720704 and 2013BAI09B03) and the National Natural Science Foundation of China (Grant number: 81241043).

Competing interests None.

Ethics approval This final study protocol (No. 201301) and written informed consent (No. 201301) have been approved by the Institutional Review Board of Beijing Tiantan Hospital Affiliated to Capital Medical University in February 2013. This amendment has been approved by the Institutional Review Board.

Provenance and peer review Not commissioned; externally peer reviewed.

Open Access This is an Open Access article distributed in accordance with the Creative Commons Attribution Non Commercial (CC BY-NC 4.0) license, which permits others to distribute, remix, adapt, build upon this work noncommercially, and license their derivative works on different terms, provided the original work is properly cited and the use is non-commercial. See: http:// creativecommons.org/licenses/by-nc/4.0/

\section{REFERENCES}

1. McNulty ML, Lee VH. Management of unruptured intracranial aneurysms and arteriovenous malformations. Am J Ther 2011;18:64-9.

2. Gross BA, Du R. Rate of re-bleeding of arteriovenous malformations in the first year after rupture. J Clin Neurosci 2012;19:1087-8.

3. Al-Shahi R, Bhattacharya JJ, Currie DG, et al. Prospective, population-based detection of intracranial vascular malformations in adults: the Scottish Intracranial Vascular Malformation Study (SIVMS). Stroke 2003;34:1163-9.

4. Andreussi L, Dhellemmes P. [Cerebral arteriovenous malformations. Natural history. Therapeutic approaches]. Agressologie 1990;31:237-9.

5. Niazi TN, Klimo P Jr, Anderson RC, et al. Diagnosis and management of arteriovenous malformations in children. Neurosurg Clin N Am 2010;21:443-56.

6. Yamada S, Brauer FS, Colohan AR, et al. Concept of arteriovenous malformation compartments and surgical management. Neurol Res 2004;26:288-300.

7. Barr JC, Ogilvy CS. Selection of treatment modalities or observation of arteriovenous malformations. Neurosurg Clin N Am 2012;23:63-75.

8. Mohr JP, Parides MK, Stapf C, et al. Medical management with or without interventional therapy for unruptured brain arteriovenous malformations (ARUBA): a multicentre, non-blinded, randomised trial. Lancet 2014;383:614-21.

9. $\mathrm{Kim} \mathrm{H}$, McCulloch CE, Johnston SC, et al. Comparison of 2 approaches for determining the natural history risk of brain arteriovenous malformation rupture. Am J Epidemiol 2010;171:1317-22.

10. Han PP, Ponce FA, Spetzler RF. Intention-to-treat analysis of Spetzler-Martin grades IV and V arteriovenous malformations: natural history and treatment paradigm. J Neurosurg 2003;98:3-7.

11. Wang S, Liu L, Zhao Y, et al. Evaluation of surgical microscope-integrated intraoperative near-infrared indocyanine green videoangiography during aneurysm surgery. Neurosurg Rev 2010;34:209-15.

12. Zhao J, Wang S, Li J, et al. Clinical characteristics and surgical results of patients with cerebral arteriovenous malformations. Surg Neurol 2005;63:156-61; discussion 61.

13. Zhao J, Yu T, Wang S, et al. Surgical treatment of giant intracranial arteriovenous malformations. Neurosurgery 2010;67:1359-70; discussion 70 .

14. Baumann SB, Noll DC, Kondziolka DS, et al. Comparison of functional magnetic resonance imaging with positron emission tomography and magnetoencephalography to identify the motor cortex in a patient with an arteriovenous malformation. J Image Guid Surg 1995;1:191-7. 
15. Stapleton SR, Kiriakopoulos E, Mikulis D, et al. Combined utility of functional MRI, cortical mapping, and frameless stereotaxy in the resection of lesions in eloquent areas of brain in children. Pediatr Neurosurg 1997;26:68-82.

16. Lazar RM, Marshall RS, Pile-Spellman J, et al. Interhemispheric transfer of language in patients with left frontal cerebral arteriovenous malformation. Neuropsychologia 2000;38:1325-32.

17. Seghier M, Lazeyras F, Momjian S, et al. Language representation in a patient with a dominant right hemisphere: fMRI evidence for an intrahemispheric reorganisation. Neuroreport 2001;12:2785-90

18. Ozdoba C, Nirkko AC, Remonda L, et al. Whole-brain functional magnetic resonance imaging of cerebral arteriovenous malformations involving the motor pathways. Neuroradiology 2002;44:1-10.

19. Cannestra AF, Pouratian N, Forage J, et al. Functional magnetic resonance imaging and optical imaging for dominant-hemisphere perisylvian arteriovenous malformations. Neurosurgery 2004:55:804-12; discussion 12-4.

20. Thickbroom GW, Byrnes ML, Morris IT, et al. Functional MRI near vascular anomalies: comparison of cavernoma and arteriovenous malformation. J Clin Neurosci 2004;11:845-8.

21. Ulmer JL, Hacein-Bey L, Mathews VP, et al. Lesion-induced pseudo-dominance at functional magnetic resonance imaging: implications for preoperative assessments. Neurosurgery 2004;55:569-79; discussion 80-1.

22. Stancanello J, Cavedon C, Francescon P, et al. BOLD fMRI integration into radiosurgery treatment planning of cerebral vascular malformations. Med Phys 2007;34:1176-84.
23. Caramia F, Francia A, Mainero C, et al. Neurophysiological and functional MRI evidence of reorganization of cortical motor areas in cerebral arteriovenous malformation. Magn Reson Imaging 2009;27:1360-9.

24. Lee L, Sitoh YY, Ng l, et al. Cortical reorganization of motor functional areas in cerebral arteriovenous malformations. $J$ Clin Neurosci 2013;20:649-53.

25. Roux FE, Boulanouar K, Ibarrola $\mathrm{D}$, et al. [Practical role of functional MRI in neurosurgery]. Neurochirurgie 2000;46:11-22.

26. Spetzler RF, Martin NA. A proposed grading system for arteriovenous malformations. J Neurosurg 1986;65:476-83.

27. Sanjuan A, Bustamante JC, Forn C, et al. Comparison of two fMRI tasks for the evaluation of the expressive language function. Neuroradiology 2010;52:407-15.

28. Pandey P, Marks MP, Harraher CD, et al. Multimodality management of Spetzler-Martin Grade III arteriovenous malformations. J Neurosurg 2012;116:1279-88.

29. van Beijnum J, van der Worp HB, Buis DR, et al. Treatment of brain arteriovenous malformations: a systematic review and meta-analysis. JAMA 2011;306:2011-19.

30. Gerganov VM, Samii A, Stieglitz L, et al. Typical 3-D localization of tumor remnants of WHO grade II hemispheric gliomas-lessons learned from the use of intraoperative high-field MRI control. Acta Neurochir (Wien) 2011;153:479-87.

31. Schaller C, Schramm J, Haun D. Significance of factors contributing to surgical complications and to late outcome after elective surgery of cerebral arteriovenous malformations. J Neurol Neurosurg Psychiatry 1998;65:547-54. 